\section{Polymerisation von Propen und Buten mit einem chiralen Zirconocen und Methylaluminoxan als Cokatalysator **}

Von Walter Kaminsky*, Klaus Külper, Hans H. Brintzinger und Ferdinand R. W. P. Wild

Mit löslichen Ziegler-Natta-Katalysatoren auf der Basis von Bis(cyclopentadienyl)zirconium(IV)-Verbindungen und Methylaluminoxan konnte bisher nur ataktisches Polypropylen hergestellt werden ${ }^{[1,2]}$. Kürzlich zeigte Ewen ${ }^{[3]}$ daß mit dem sterisch starren [Ethylenbis(-1-indenyl)]titandichlorid (Gemisch aus Mesoform und Racemat) ${ }^{[4]}$ Gemische aus z. B. $63 \%$ isotaktischem und $37 \%$ ataktischem Polypropylen entstehen.

Wird jedoch das chirale [Ethylenbis(4,5,6,7-tetrahydro1-indenyl)]zirconiumdichlorid 1 (Abb. 1) und als Cokatalysator Methylaluminoxan der Struktur $\left[\mathrm{Al}\left(\mathrm{CH}_{3}\right)-\mathrm{O}\right]_{n}$ verwendet, so erhält man hoch isotaktisches Polypropylen. Für diese Polymerisation wurde das Racemat von 1 eingesetzt. Der in Toluol lösliche Anteil kann auf 0.2 Gew.-\% gesenkt werden und liegt damit noch erheblich unter den Werten (2-7\%), die mit heterogenen Katalysatoren auf der

[*] Prof. Dr. W. Kaminsky, Dipl--Chem. K. Külper Institut für Technische und Makromolekulare Chemie der Universităt Martin-Luther-King-Platz 6, D-2000 Hamburg 13

Prof. Dr. H. H. Brintzinger, Dr. F. R. W. P. Wild Fakultăt für Chemie der Universităt Postfach 5560, D-7750 Konstanz 1

[**] Diese Arbeit wurde von der Deutschen Forschungsgemeinschaft unterstutzt. Wir danken der Hoechst AG für Hilfe bei der Polymeranalytik.
Basis von $\mathrm{TiCl}_{4} / \mathrm{MgCl}_{2} / \mathrm{Al}\left(\mathrm{C}_{2} \mathrm{H}_{5}\right)_{3} / \mathrm{C}_{6} \mathrm{H}_{5} \mathrm{CO}_{2} \mathrm{C}_{2} \mathrm{H}_{5}$ erreichbar sind ${ }^{[6]}$.

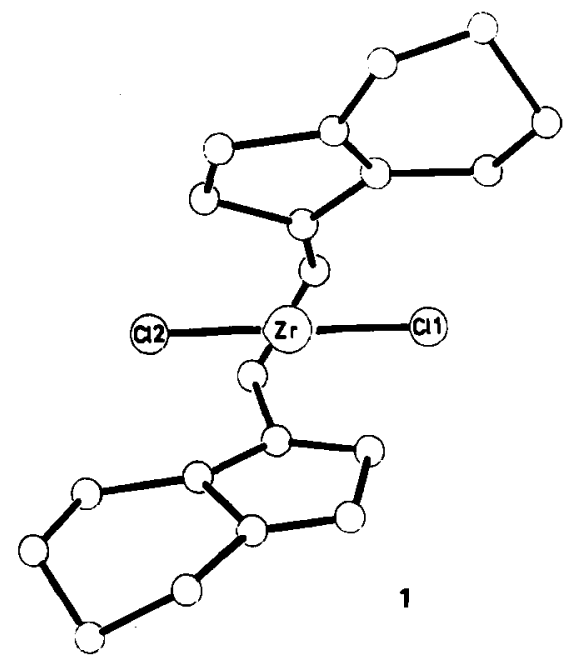

Abb. 1. Struktur von chiralem $\left[\mathrm{C}_{2} \mathrm{H}_{4}(4,5,6,7-\text { Tetrahydro-1-indenyl })_{2} \mathrm{ZrCl}_{2}\right] 1$ [5] im Kristall.

Erstaunlich ist die hohe Aktivität des chiralen Katalysators 1 (Tabelle 1). So genügen davon weniger als $10^{-5}$ $\mathrm{mol} / \mathrm{L}$, um bei $60^{\circ} \mathrm{C}$ in der Stunde $7700 \mathrm{~kg}$ Polypropylen pro mol $\mathrm{Zr} \mathrm{zu}$ erzeugen.

Tabelle 1. Polymerisation von Propen oder Buten mit 1 und Methylaluminoxan. Bedingungen: $8.4 \cdot 10^{-6} \mathrm{~mol} / \mathrm{L}$ rac- 1 in $330 \mathrm{~mL}$ Toluol, $70 \mathrm{~mL} \alpha$-Olefin, $1.6 \cdot 10^{-2} \mathrm{~mol} / \mathrm{L} \mathrm{Al}$-Einheiten von Methylaluminoxan $\left(M_{\eta} 1.200\right)$ bei unterschiedlichen Temperaturen (Pol. $=$ Polymer, $M_{\eta}=$ mittleres Molekulargewicht).

\begin{tabular}{|c|c|c|c|c|c|}
\hline$\alpha$-Olefin & $\begin{array}{c}T \\
{\left[{ }^{\circ} \mathrm{C}\right]}\end{array}$ & $\begin{array}{l}t \\
{[\mathrm{~min}]}\end{array}$ & $\begin{array}{l}\text { Ausb. } \\
\text { [g] }\end{array}$ & $\begin{array}{l}\text { Aktivität } \\
{\left[\frac{\mathrm{kg} \text { Pol. }}{\mathrm{mol} \mathrm{Zr} \cdot \mathrm{h}}\right]}\end{array}$ & $\boldsymbol{M}_{\eta}$ \\
\hline Propen & -20 & 360 & 1.5 & 80 & 300000 \\
\hline Propen & -10 & 270 & 4.5 & 300 & 280000 \\
\hline Propen & 0 & 255 & 12.5 & 880 & 130000 \\
\hline Propen & 8 & 180 & 13.0 & 1300 & 85000 \\
\hline Propen & 15 & 170 & 26.7 & 2900 & 55000 \\
\hline Propen & 20 & 120 & 31.3 & 4750 & 41000 \\
\hline Propen & 60 & 90 & 38.7 & 7700 & 800 \\
\hline 1-Buten & -10 & 330 & 9.1 & 500 & 150000 \\
\hline 1-Buten & +20 & 200 & 29.2 & 2640 & 50000 \\
\hline
\end{tabular}

Unter ähnlichen Bedingungen werden mit Bis(cyclopentadienyl)dimethylzirconium in der Stunde nur $2730 \mathrm{~kg}$ ataktisches Polypropylen pro mol $\mathrm{Zr}$ gebildet. Das chirale Zirkonocen 1 liefert also nicht nur isotaktisches Polypropylen, sondern hat auch eine um den Faktor 2-3 größere Polymerisationsaktivität als Katalysatoren, die nur ataktisches Polypropylen bilden. Während sich die Polymerisationsgeschwindigkeiten von Ethylen : Propen mit Bis(cyclopentadienyl)zirconium-Verbindungen wie $25: 1$ verhalten, sinkt dieses Verhältnis hier auf ca. $10: 1^{[7,8]}$. Dies bedeutet, daß Propen vom Zirconocen 1 relativ besser polymerisiert wird als von Bis(cyclopentadienyl)zirconiumVerbindungen.

Neben der hohen Aktivităt von 1 gegenüber Propen, die mindestens die Werte von heterogenen Katalysatoren erreicht, sind auch die Eigenschaften der gebildeten Polypropylene bemerkenswert (Tabelle 2). Im Gegensatz zu technischem Polypropylen, das eine Molekulargewichtsverteilung $M_{\mathrm{w}} / M_{\mathrm{n}}$ von minimal 5 aufweist, haben die mit 1 
Tabelle 2. Zusammensetzung von mehreren, mit 1 hergestellten Polypropylenen (siehe Tabelle 1).

\begin{tabular}{rrllll}
\hline$M_{\eta}$ & $M_{\mathrm{w}}$ & $\begin{array}{l}M_{\mathrm{w}} / M_{\mathrm{n}} \\
{[\mathrm{a}]}\end{array}$ & $\begin{array}{l}\text { atakt. } \\
\text { Antei] } \\
{[\%][\mathrm{b}]}\end{array}$ & $\begin{array}{l}\text { Isotaktizitäts- } \\
\text { Index } \\
{[\%][\mathrm{c}]}\end{array}$ & $\begin{array}{l}\text { Viskosităts- } \\
\text { zah] } \\
{\left[\mathrm{cm}^{3} / \mathrm{g}\right] \text { [d] }}\end{array}$ \\
\hline 280000 & 305000 & 2.6 & 0.25 & 91 & 242 \\
130000 & 144000 & 2.4 & 0.2 & 88.1 & 132 \\
55000 & 62000 & 2.0 & 0.7 & 87.3 & 110 \\
41000 & 45000 & 1.9 & 1.0 & 86.0 & 76 \\
\hline
\end{tabular}

[a] GPC-Messungen bei $135^{\circ} \mathrm{C}$ in Trichlorbenzol. [b] In gesåttigten Kohlenwasserstoffen $\left(\mathrm{Kp}=130^{\circ} \mathrm{C}\right)$ löslicher Anteil nach Kloos et al. [9]. [c] IR-spektroskopisch nach Luongo [10]. [d] Bei $135^{\circ} \mathrm{C}$ in Decalin.

erzeugten Produkte Werte von 1.9 bis 2.6. Der in Kohlenwasserstoffen lösliche Anteil liegt bei allen Proben höchstens bei $1 \%$. Der IR-spektroskopisch bestimmte Isotaktizitätsindex ist mit 86 bis $91 \%$ ebenfalls sehr hoch. Die Kristallinităt des hoch isotaktischen Polypropylens wird von der Einheitlichkeit der Stereochemie der Polymerisation geprägt. Die hochauflösende ${ }^{13} \mathrm{C}-\mathrm{NMR}$-Spektroskopie gibt Einblick in den Aufbau der Sequenzen in der Polymerkette. Für die isotaktischen Sequenzen (mm-Triaden) ergeben sich Werte von $95.9 \%$, für die heterotaktischen (mr-Triaden) Werte von 3.2\% und für die syndiotaktischen (rr-Triaden) Werte von $0.9 \%$. Auffällig ist der sehr geringe Anteil an rr- im Vergleich zu den mr-Triaden. Bei handelsüblichem Polypropylen hat das Verhältnis mr/rr die Größenordnung 1, und rr ist um den Faktor 5 größer.

Mit diesem homogenen Ziegler-Natta-Katalysator werden also Propenmoleküle hochgradig stereospezifisch inseriert. Fehler beim Einbau bleiben singulär, was durch die hohe Isotaxie von $97.5 \%$ und eine mittlere Länge der isotaktischen Sequenzen von über 60 Monomereinheiten bestätigt wird.

Auch 1-Buten läßt sich mit dem Katalysator aus dem Zirconocen 1 und Methylaluminoxan mit hohen Aktivitäten polymerisieren (vgl. Tabelle 1). So beträgt die Polymerisationsaktivität bei $20^{\circ} \mathrm{C}$ in der Stunde noch $2640 \mathrm{~kg}$ Polybuten pro mol Zr. Obwohl die Polymerisate mittlere Molekulargewichte von 150000 oder 50000 haben, sind sie in Toluol löslich. Die Konsistenz ist wachsartig bis kristallin, nicht aber klar und viskos wie bei ataktischem Polybuten, das mit Bis(cyclopentadienyl)zirconium-Verbindungen gewonnen und ${ }^{13} \mathrm{C}$-NMR-spektroskopisch vermessen wurde.

Mit 1 und Methylaluminoxan kann somit sowohl ungewöhnlich hoch isotaktisches Polypropylen als auch isotaktisches Polybuten hergestellt werden; die Aktivität des Katalysators ist hoch. Die enge Molekulargewichtsverteilung, ein zusätzlicher Effekt, dürfte verarbeitungstechnisch von großem Interesse sein.

\section{Arbeitsvorschrift}

1: In einen Dreihalskolben werden bei $-196^{\circ} \mathrm{C}$ unter Rüren $60 \mathrm{~mL}$ Tetrahydrofuran (THF) einkondensiert und mit $4.9 \mathrm{~g}$ ( $21 \mathrm{mmol}) \mathrm{ZrCl}_{4}$ versetzt. Unter $\mathrm{N}_{2}$-Schutz werden dazu bei Raumtemperatur 21 mmol des Dilithiumsalzes von Bis(1-indenyl)ethan in $50 \mathrm{~mL}$ THF getropft. Nach $2 \mathrm{~h}$ Rühren wird Chlorwasserstoff eingeleitet und sofor danach der ÜberschuB im Vakuum entfernt. Die Mischung wird dann mit $20 \mathrm{~mL}$ Diethylether und $10 \mathrm{~mL}$ Petrolether versetzt. Das hellgelbe, kristalline Produkt wird nacheinander mit $4 \mathrm{~N}$ Salzsäure, Ethanol und Diethylether gewaschen und im Vakuum getrocknet; Ausbeute $3.1 \mathrm{~g}(R, S)-\left[1,1^{\prime}\right.$-Ethylenbis(indenyl)]zirconiumdichlorid. $1 \mathrm{~g}$ dieser Substanz wird entsprechend der Vorschrift [4] für die analoge Titanverbindung 30 min unter Zugabe von $75 \mathrm{mg} \mathrm{PtO}$ und $25 \mathrm{~mL} \mathrm{CH}_{2} \mathrm{Cl}_{2}$ bei 100 bar $\mathrm{H}_{2}$ hydriert: Ausbeute $650 \mathrm{mg} 1$ (charakterisiert durch Rontgen-Strukturanalyse [5]).

Polymerisation von Propen: In einem 1L-Glasautoklaven mit Thermostat und Magnetrührer werden nach Ausheizen und Spälen mit Ar $330 \mathrm{~mL}$ Toluol vorgelegt, in die $320 \mathrm{mg}(5.6 \mathrm{mmol} \mathrm{Al})$ Methylaluminoxan und $3.3 \cdot 10^{-6} \mathrm{~mol}$
1 in $1 \mathrm{~mL}$ Toluol gegeben werden. Nach $20 \mathrm{~min}$ Alterungszeit werden $70 \mathrm{~mL}$ gereinigtes Propen einkondensiert, und der Inhalt wird auf $20^{\circ} \mathrm{C}$ thermostatisiert. Wenig später făllt weißes Polypropylen aus. Nach 120 min setzt man Ethanol zu, filtrier, wäscht mit verdünnter $\mathrm{HCl}$ und trocknet im Vakuum; Ausbeute $31.3 \mathrm{~g}$ isotaktisches Polypropylen. Im toluolischen Filtrat bleibt nach Abziehen des Lơsungsmittels ein Rückstand von $0.3 \mathrm{~g}$ Polypropylen.

[1] H. Sinn, W. Kaminsky, Adv. Organomet. Chem. 18 (1980) 99.

[2] W. Kaminsky, M. Miri, H. Sinn, R. Woldt, Makromol. Chem. Rapid Commun. 4 (1983) 417.

[3] J. A. Ewen, J. Am. Chem. Soc. 106 (1984) 6355.

[4] F. R. W. P. Wild, L. Zsolnai, G. Huttner, H. H. Brintzinger, J. Organomet. Chem. 232 (1982) 233.

[5] F. R. W. P. Wild, M. Wasiucionek, G. Huttner, H. H. Brintzinger, J. Organomet. Chem., im Druck.

[6] P. Pino, R. Mulhaupt, Angew. Chem. 92 (1980) 869; Angew. Chem. Int. Ed. Engl. 19 (1980) 857.

[7] H. Sinn, W. Kaminsky, H.-J. Vollmer, R. Woldt, Angew. Chem. 92 (1980) 396: Angew. Chem. Int. Ed. Engl. 19 (1980) 390.

[8] W. Kaminsky, Naturwissenschaften 71 (1984) 93.

[9] F. Kloos, H. G. Lengering, IUPAC Macro Florence, Reprints 2 (1980) 479.

[10] J. P. Luongo, J. Polym. Sci. 42 (1960) 139. 